\title{
Novel image processing interface to relate DSB spatial distribution from experiments with phosphorylation foci to the state-of-the-art models of DNA breakage
}

\author{
Artem L. Ponomarev ${ }^{\mathrm{a}, \mathrm{b}, *}$, Francis A. Cucinotta ${ }^{\mathrm{b}}$ \\ ${ }^{a}$ USRA, 3600 Bay Area Blvd., Houston, TX 77058, USA \\ b NASA Lyndon B. Johnson Space Center, 2101 NASA Parkway, SK, Houston, TX 77058, USA
}

Received 1 June 2005; accepted 10 November 2005

\begin{abstract}
We apply new image processing tools and computer modeling of DSB formation to analyze new experimental data on DSB (DNA doublestrand break) yield (\#DSB per base pair per Gray). There is LET-dependent DSB clustering within the nucleus volume, and clustering of DSBs along the DNA length, which we show through modeling the whole set of human chromosomes. In recent experiments, DSBs are imaged as phosphorylation sites of the histone protein $\mathrm{H} 2 \mathrm{AX}$, denoted as $\gamma \mathrm{H} 2 \mathrm{AX}$ foci, and it is suggested that foci images indicate the spatial distribution of DSBs. For high-LET radiation, DSBs should be located closer to the track center or grouped around ionization sites leading to clustered DSBs as described theoretically, and as can be seen in images. We describe the successful segmentation of foci images and determine foci statistics that will relate models of DSB spatial correlation and clustering along DNA length to the experimental data. The foci data can be used to analyze high-LET effects on DNA fragment sizes and DSB distributions.
\end{abstract}

(C) 2006 Elsevier Ltd. All rights reserved.

Keywords: Phosphorylation foci; DNA damage; Random walks; Image segmentation; DNA fragments; Double-strand breaks

\section{Introduction}

The comparison of computational DNA breakage models to experiments measuring DNA damage using pulsed-field gel electrophoresis (PFGE) or more recently based on imaging of phosphorylation foci of H2AX histone is complicated by possible limitations in the theoretical description of DNA structure and potential limitations in spatial resolution of such experimental techniques. The analysis of $\gamma \mathrm{H} 2 \mathrm{AX}$ foci (MacPhail et al., 2003) will require new statistical approaches and image processing tools. A main goal of computational models is to determine the number and spatial distribution of DSBs per DNA base pair per Gray for various radiations (high and

\footnotetext{
* Corresponding author. NASA JSC, SK, 2101 NASA Parkway, build. 37, \#119, Houston, TX 77058, USA. Tel.: +1 281483 0089; fax: +1 8662886491. E-mail address: aponomar@ems.jsc.nasa.gov (A.L. Ponomarev).
}

low LET). Methods currently in use to measure DNA breakage are still suffering from limitations on how to determine the DSB yield measured from raw experimental data, as it requires some modeling, the critique of which we will present here. One limitation is the absence of a consistent theoretical model applied to the data analysis, which includes the geometry of high-LET tracks, and DNA fibers, chromosomes and the cell nucleus. In this paper we will consider several aspects of current experimental and theoretical approaches of the measurement and theoretical prediction of DSB statistics.

In the PFGE experiment (Stenerlöw et al., 2003; Löbrich et al., 1996; Höglund et al., 2000), for example, data for small fragments are absent at a certain small scale due to limitations inherent in the experimental technique. The DSB yield can only be obtained from the fragment-size distribution function for all sizes, from a few bp to Mbp. Thus, some DNA breakage modeling is used for extrapolation to small sizes. Such modeling needs to account for high-LET effects in some cases (such as 
HZE particles) to refine the obtained yields. Our efforts on this refinement follow in the results section.

Another important aspect affecting the accuracy of DSB yield estimates in PFGE experiments is the subtraction of background DNA fragments at 0 Gray. Some background signal, detected in the control cells, corresponds to DNA fragmentation present in the system without radiation. We believe that correct subtraction of the background signal cannot be obtained by merely subtracting readings in the corresponding fragment size bins. In a nutshell, a better approach is to treat the resulting histogram obtained from the experiment as a superposition of point processes (Sachs et al., 1999), which arises from the DSB placement process and some unknown background process. This superposition is non-linear and the subtraction of the background process from the fragments produced by radiation can be developed analytically. Using the language of an advanced probabilistic technique (Sachs et al., 1999), the DSB process due to radiation is given by a functional equal to the observed process (reported experimental data) functional divided by the background process functional (and divided by the telomere process functional, which is a less significant correction for high doses). In addition to this required calibration of the signal, the extrapolation of PFGE data to the fragments of small size cannot be done using a random breakage model based on Poisson process for high LET. Instead, the model for non-random breakage for high-LET radiation should be used (Ponomarev et al., 2001). The theoretical work on the background subtraction is underway and will be published elsewhere.

New experiments with foci corresponding to phosphorylation sites of proteins like $\gamma \mathrm{H} 2 \mathrm{AX}$ or ATM, offer a novel way to attack the problem of DSB count and their spatial distribution, since the foci count per cell relates to the DSB yield as a function of dose. This new technique requires powerful image processing tools, which we will discuss further. It is important to be aware that the more ubiquitous two-dimensional images of foci have the problem of oversaturation (overlap in $z$ direction) which is a consequence of the fact that total light intensity for each pixel in the image is recorded from a certain depth interval. Confocal microscopy might resolve the problem of the overlap. For now, we need to take into account the foci "eclipse," that is, the visible overlap in a 2-d image of closely located foci in $(x, y, z)$. A simple model that adjusts the number of visible foci is given by

$m_{\mathrm{a}}=m\left(1+\frac{A m}{S}\right)$,

where $m_{\mathrm{a}}$ is the adjusted focus count in an image of area $S, m$ is the number of visible foci, $A$ is an average area of a focus ( $A m \ll S$ is assumed).

The problem of subtracting the background and relating the visible foci to DSBs remains. The background density of foci is high, so it cannot be assumed to be negligible to the number of foci due to radiation. In fact, the background foci can be quite numerous and dependent on the cell cycle (MacPhail et al., 2003). If two foci overlap, they can either both be caused by radiation, or both belong to the background, or one can be due to radiation and the other due to the background. This work on background subtraction and the other work on relating the number of foci to the fragment-size distribution function simulated by our model, which is based on the random walk geometry of chromosomes and the detailed track structure, are underway.

The previously developed DNAbreak model (Ponomarev et al., 2001) was created for the whole genome and for large fragments on the Mbp scale. A random walk model for chromosomes and an averaged track structure for energy depositions were used. This model successfully predicted the distribution of large DNA fragments and indicated how this distribution can be related to the experimental data, as well as how the model can be extrapolated to lower doses and smaller fragment sizes. The most important conclusion was that we only need one adjustable parameter, $\boldsymbol{Q}$, to predict the spatial and fragment-size distributions, as well as the number of DSBs per bp per Gray (Ponomarev et al., 2001). This parameter is the "true" DSB yield, and is a refinement of yields reported in recent experimental papers. We determined this parameter by fitting PFGE data with our model to produce the best upto-date estimate. We showed that it weakly depends on LET (Table 1), with the conclusion that all the peculiarity in DSB distribution resulting from high LET, namely clustering both in space and along DNA, are due to the superposition of DNA geometry and track geometry. This model allowed us to separate two physical processes: a local process of DSB formation from a certain amount of incident energy in a small volume, as characterized by LET-insensitive parameter $\boldsymbol{Q}$; and a global process of many DSBs and correlations among them, with high sensitivity to LET values, as determined by DNA and track geometry on a large scale. The DNAbreak model also determined the cumulative probability of all fragment sizes below approximately $3 \mathrm{kbp}$, which was a workaround for the problem of small fragment sizes in PFGE experiments. The model could not predict the exact distribution for fragment sizes below this cutoff limit, but the DSB yield followed from the cumulative distribution determined with higher precision.

Here we present our refinement of DSB yields for highLET radiations and preliminary results of phosphorylation foci statistics.

\section{Materials and methods}

\subsection{Cell preparation}

Cell preparation was as described in the paper by Desai et al. (2005). In our experiments we used normal human fibroblasts (AG1522 from NIA cell repository) and a primary antibody (anti- $\gamma$-H2AX polyclonal, Upstate Biotechnology). Cells were analyzed with a Ziess Axioplan fluorescence microscope. Images were captured using a CCD camera and Mac Probe analysis software from Applied Imaging.

\subsection{Image segmentation}

The Windows platform was used for DNA and particle track simulations. We used the NeuronMapper ${ }^{\mathrm{TM}}$ (copyright 


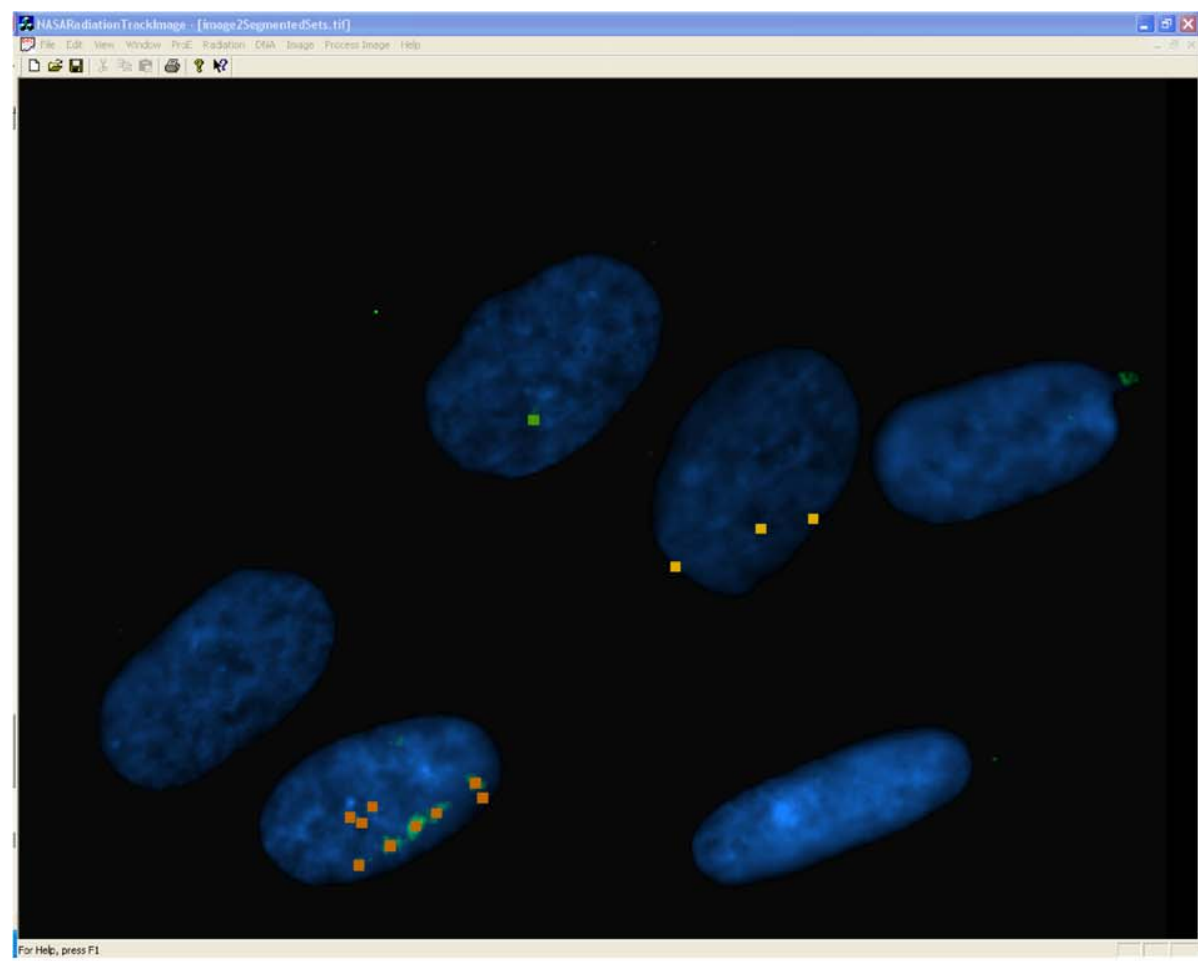

(a)

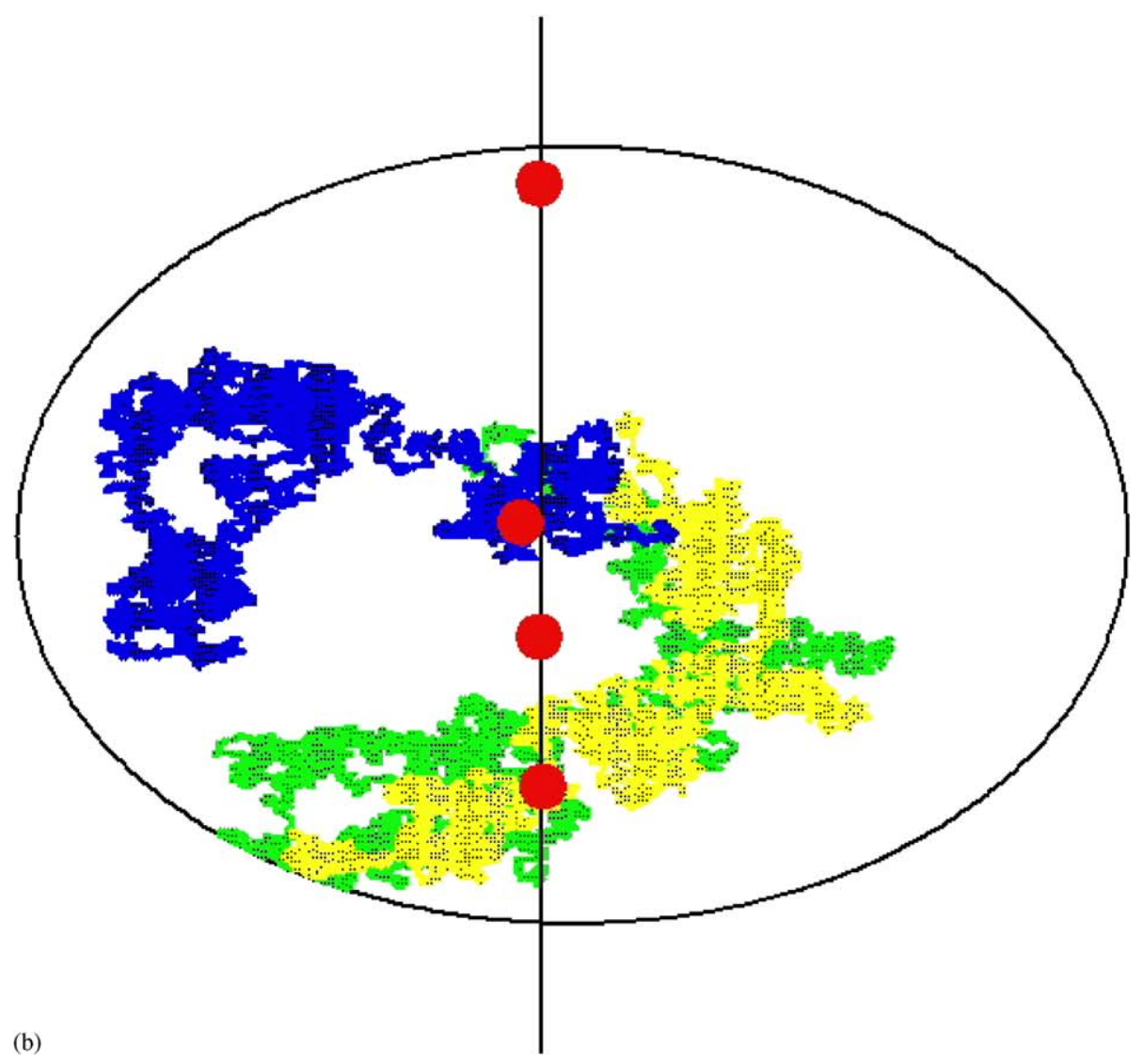

Fig. 1. New experimental and model data. Panel A. A snapshot of a few human fibroblasts and $\gamma \mathrm{H} 2 \mathrm{AX}$ foci at $0.2 \mathrm{~Gy}$ and $1 \mathrm{~h}$ after $1 \mathrm{GeV}$ titanium ion radiation. Foci and cell nuclei are segmented and counted (individual colors in squares represent foci belonging to a particular cell). Panel B. A schematic human cell plated to a substrate is modeled as an ellipsoid. All human chromosomes are modeled. For clarity, the three smallest chromosomes are shown in color. Their configuration is given by a random walk. The center of a track is shown as a vertical line. In this single Monte Carlo realization several DSBs are produced close to the track center (red dots). 
Table 1

Track efficiency parameter

\begin{tabular}{|c|c|c|c|c|c|c|c|}
\hline$Q \times 10^{5}$ & $\begin{array}{l}\mathrm{Fe}, 150 \\
(\mathrm{keV} / \mu \mathrm{m})\end{array}$ & $\begin{array}{l}\mathrm{N}, 80-97 \\
(\mathrm{keV} / \mu \mathrm{m})\end{array}$ & $\begin{array}{l}\mathrm{N}, 125 \\
(\mathrm{keV} / \mu \mathrm{m})\end{array}$ & $\begin{array}{l}\mathrm{N}, 175 \\
(\mathrm{keV} / \mu \mathrm{m})\end{array}$ & $\begin{array}{l}\mathrm{N}, 225 \\
(\mathrm{keV} / \mu \mathrm{m})\end{array}$ & $\begin{array}{l}\mathrm{He}, 40 \\
(\mathrm{keV} / \mu \mathrm{m})\end{array}$ & $\begin{array}{l}{ }^{60} \text { Co-photons }<0.5 \\
(\mathrm{keV} / \mu \mathrm{m})\end{array}$ \\
\hline $150-200 \mathrm{~Gy}$ & $3.71 *$ & $2.46^{*}, 1.70$ & 1.81 & 1.43 & 1.41 & 1.83 & 1.2 \\
\hline $100 \mathrm{~Gy}$ & & 1.72 & 1.78 & 1.56 & 1.46 & 1.67 & 1.2 \\
\hline $50-80 \mathrm{~Gy}$ & $3.87 *$ & $2.49 *, 1.64$ & $1.78,1.81$ & 1.44 & 1.47 & & $1.08,1.25$ \\
\hline $30 \mathrm{~Gy}$ & & & 1.78 & & & & 1.25 \\
\hline
\end{tabular}

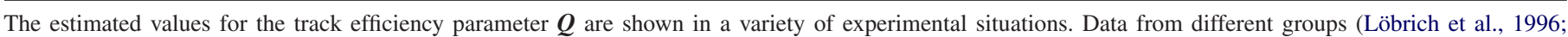

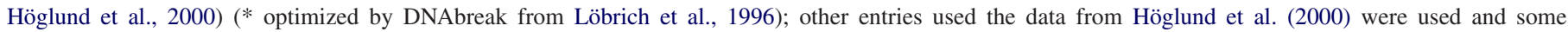

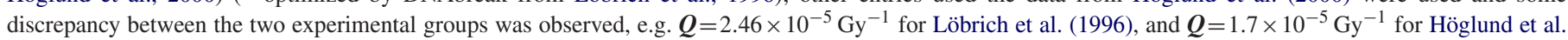

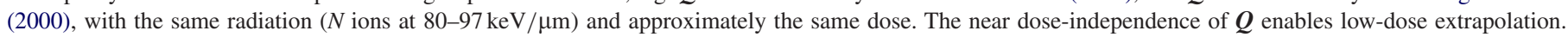

by BCM, Texas Medical Center, Houston, TX, USA) graphical users interface (GUI), which is written is Visual $\mathrm{C}++$ 6.0. The program used for DNA simulation, DNAbreak, was combined with Visual FORTRAN code previously employed by Cucinotta et al. (1998). These tools are now combined into one master interface called NASARadiationTrackImage ${ }^{\mathrm{TM}}$ (copyright by USRA, Houston, TX, USA).

\section{Results}

\subsection{Image analysis}

The segmentation of the experimental image data was performed with NeuronMapper ${ }^{\mathrm{TM}}$, which was originally created to map Drosophila fly brain (Ponomarev and Davis, 2003). As a derivative of this technology, we developed a function that can independently perform object segmentation in RGB channels and then cross-examine the results of this segmentation. The foci (given in the green channel) are counted only if they overlap the corresponding nuclei (given in the blue channel). This count provides the number of foci per cell per Gray. Through this procedure we determined the statistics of foci per nucleus. We obtained the total number of foci per nucleus, average intensity and average area. The results for human fibroblasts, $\gamma \mathrm{H} 2 \mathrm{AX}$ phosphorylation sites at $1 \mathrm{~h}$ after $1 \mathrm{GeV}$ titanium ion radiation at a dose of $0.2 \mathrm{~Gy}$ are the following: the average light intensity per focus is 1287 dimensionless units (here 255 is the maximum allowed intensity in the image per pixel). The average area of foci in pixel squared (pixel linear dimension is $\approx 0.1 \mu \mathrm{m})$ is 8.86 pixels, or about $0.089 \mu \mathrm{m}^{2}$. The number of foci per cell on average is 2.23 . We used this image (Fig. 1A) to show the capabilities of our program. These results will be extrapolated to many images at different doses to find the number of DSBs per Gy per cell.

\subsection{Up-to-date estimates}

The new image data can be related to the data of the previously described model (Ponomarev et al., 2001), which generalizes models of DSB placement along DNA based on a theory of random clusters of DSBs that takes into account the chromosome geometry and the track structure. It is known that chromosomes are randomly coiled most of the time (during interphase, for instance), so the random walk model is suitable in the majority of cases. More thorough geometrical models of DNA should take into account the self-avoidance of chromatin and the boundaries of the nucleus. We already have such preliminary results (Fig. 1B). Another important feature of our approach is the use of the radial distribution of energy as the function of the ion's charge and velocity. In a track the energy profile is usually sharp, leading to DSB placement roughly along a line within a cell. When juxtaposed onto the random chromosome geometry, this track structure results in the socalled non-random DSB distribution (Ponomarev et al., 2001). This model can be fitted to numerous PFGE experimental data (Löbrich et al., 1996; Höglund et al., 2000). In the model we assume that only one parameter is unknown, the microscopic DSB yield $\boldsymbol{Q}$, or the number of DSBs per small physical volume per Gy. The data for $\boldsymbol{Q}$ as obtained from PFGE experiments is given in Table 1. We should acknowledge that though conceptually correct, these results are numerically tentative, because of the previously mentioned challenge with background subtracting. The improvement of these results will require more close collaboration with experimentalists. We believe this subtraction needs to be done in the framework of our randomly located-clusters approach. The correct results can be obtained by removing the background chromosome breakage process, which is equivalent to the elimination of the background point process and the telomere point process from the observed radiation breakage process as described in Sachs et al. (1999).

\section{Discussion}

Computational models that include detailed radiation track structure and the random geometry of DNA will provide the radiobiological community with a valuable and robust tool. There is much work to be done before this goal is achieved. We believe that the proper interpretation of experimental data cannot be done without an accurate theoretical approach as we outlined earlier. New experimental data for phosphorylation foci are very encouraging, as this method will present an alternative way to test theoretical models, which in the past have relied on methods such as PFGE applicable only at high doses. We believe the main problem in all experimental data is the proper subtraction of the noise, or background signal. This subtraction must be done properly in order to define the fragment-size distribution function, which is related to DSB yields. In all cases 
the function will only be defined above a certain fragment size limit. This resolution limit, measured in DNA molecular mass or base pairs, is different in different models. In our randomly located-clusters models it is about $3 \mathrm{kbp}$. Other models have more detailed DNA structure in the range of 10-100 bp. To be correct, various models should be consistent for the prediction of the fragment-size distribution at a certain size common to them. We propose to merge these models, such as DNAbreak and the Nikjoo model (Nikjoo et al., 2002) at the scale of $3 \mathrm{kbp}$.

The ultimate purpose will be to reconcile the DSB yield estimates by both experiment and theory. Only when these are consistent can we claim that the experimental signal is properly interpreted, with the correct resulting DSB yields and theoretical data.

\section{Acknowledgments}

Funding was through NASA under the Risk Assessment Project. Special thanks to Dr. Honglu Wu for image data, and K. George and N. Desai for clarifying experimental methods.

\section{References}

Cucinotta, F.A., Katz, R., Wilson, J.W., 1998. Radial distribution of electron spectra from high-energy ions. Radiat. Environ. Biophys. 37 (4), 259-265.
Desai, N., Durante, M., Lin, Z.W., Cucinotta, F.A., Wu, H., 2005. High-LETinduced $\mathrm{H} 2 \mathrm{AX}$ phosphorylation around the Bragg curve. Adv. Space Res. 35 (2), 236-242.

Höglund, E., Blomquist, E., Carlsson, J., Stenerlöw, B., 2000. DNA damage induced by radiation of different linear energy transfer: initial fragmentation. Int. J. Radiat. Biol. 76 (4), 539-547.

Löbrich, M., Cooper, P.K., Rydberg, B., 1996. Non-random distribution of DNA double-strand breaks induced by particle irradiation. Int. J. Radiat. Biol. 70, 493-503.

MacPhail, S.H., Banath, J.P., Yu, T.Y., Chu, E.H.M., Lambur, H., Olive, P.L., 2003. Expression of phosphorylated histone $\mathrm{H} 2 \mathrm{AX}$ is cultured cell lines following exposure to X-rays. Int. J. Radiat. Biol. 79, 351-358.

Nikjoo, H., Bolton, C.E., Watanabe, R., Terissol, M., O’Neill, P., Goodhead, D.T., 2002. Modeling of DNA damage induced by energetic electrons (100 eV-100 keV). Radiat. Prot. Dosimetry 99 (1-4), 77-80.

Ponomarev, A.L., Davis, R.L., 2003. An adjustable-threshold algorithm for the identification of objects in three-dimensional images. Bioinformatics 19 (11), 1431-1435.

Ponomarev, A.L., Cucinotta, F.A., Sachs, R.K., Brenner, D.J., Peterson, L.E., 2001. Extrapolation of the DNA fragment-size distribution in a high-dose PFGE assay to low doses. Radiat. Res. 156 (5), 594-597.

Sachs, R.K., Hahnfeldt, P.G., Ponomarev, A.L., Hlatky, L.R., 1999. Largescale clustering on chromosomes of double strand breaks produced at high LET: estimates based on random walk chromatin mode. Math. Biosci. 159, 165-187.

Stenerlöw, B., Karlsson, K.H., Cooper, B., Rydberg, B., 2003. Measurement of prompt DNA double-strand breaks in mammalian cells without including heat-labile sites: results for cells deficient in nonhomologous end joining. Radiat. Res. 159, 502-510. 